\title{
5 Weiterentwicklung internationaler Terminologien und Klassifikationen und ihre Anwendung in Deutschland am Beispiel der ICD
}

\author{
Stefanie Weber
}

Im Laufe des letzten Jahrhunderts hat die Medizin nahezu täglich große Fortschritte gemacht. Von dem zufälligen Entdecken des Penicillins bis zur vollständigen Kartierung des menschlichen Genoms waren es nur wenige Jahrzehnte. Auch die kommunikative Vernetzung der Menschheit und die Geschwindigkeit des Informationsaustausches haben sich enorm weiterentwickelt. Der herkömmliche handgeschriebene Brief, der wochenlang per Schiff über den Atlantik befördert wurde, ist durch die Möglichkeiten der Übermittlung von Informationen über das Internet innerhalb von Sekunden nahezu obsolet geworden. Der Austausch von Informationen ist heute weltweit, schnell und in großem Umfang möglich.

Im Zuge dieses Fortschrittes hat sich die Weiterentwicklung von medizinischen Klassifikationen und Terminologien ebenfalls stark verändert. Ursprünglich für die Gruppierung von einigen wenigen Todesursachen geschaffen, dann zum „internationalen Krankheitsverzeichnis“ weiterentwickelt (s. Abb. 1) und heute als „Internationale statistische Klassifikation der Krankheiten und verwandter Gesundheitsprobleme" in zehnter Revision (ICD-10) [WHO, 2010] im Einsatz hat die bekannteste Klassifikation im Gesundheitswesen einen ebenso deutlichen Wandel erfahren.

\subsection{Anwendung der ICD-10 in Deutschland}

Seit der Veröffentlichung der ersten Fassung der ICD-10 nach der Verabschiedung durch die World Health Assembly sind schon 20 Jahre vergangen. In dieser Zeit ist die Implementierung der ICD-10 in Deutschland erfolgt, und zwar 
B Der fachliche Hintergrund: Terminologien und Ordnungssysteme in der

Patientenversorgung, medizinischen Forschung und Gesundheitswirtschaft
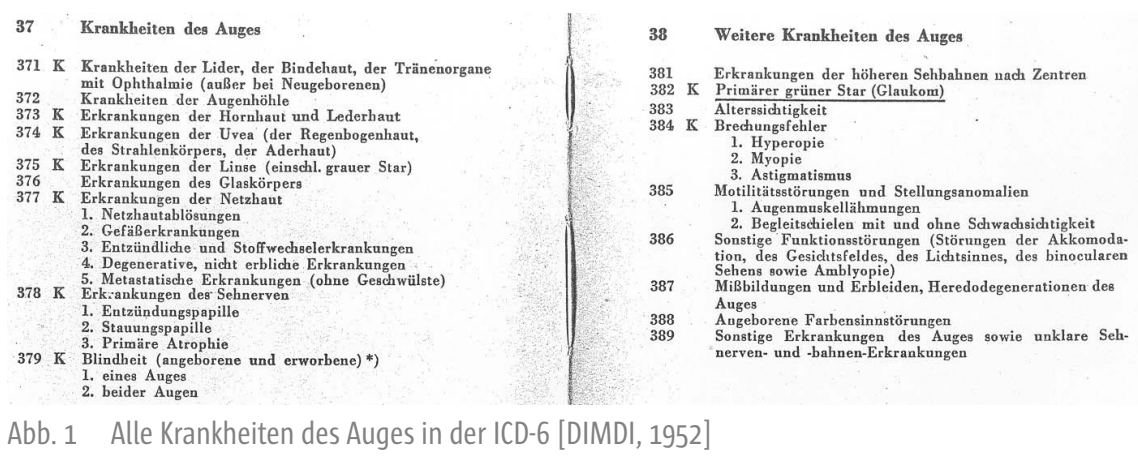

Abb. 1 Alle Krankheiten des Auges in der ICD-6 [DIMDI, 1952]

in weit größerem Maße, als das bei den Vorgängerrevisionen der Fall war [DIMDI, 2013]. Neben der deutschsprachigen Übersetzung der ICD-1o der WHO, der sog. ICD-10-WHO, gibt es in Deutschland eine zweite Fassung: Nach und nach wurde die ICD-10 an die Belange des deutschen Gesundheitswesens angepasst und modifiziert zur ICD-10-GM. Die ICD-10-GM wird zurzeit jährlich aktualisiert, sie ist in vielen Bereichen des deutschen Gesundheitssystems im Einsatz und fester Bestandteil vieler IT-Systeme im Gesundheitswesen.

Die Anwendungsfelder der ICD-10 sind vielfältig, im Folgenden werden einige wenige Beispiele genannt.

\subsubsection{Todesursachen}

Die deutschsprachige Übersetzung der Originalfassung der ICD-1o in der jeweils aktuellen Fassung, die ICD-10-WHO, wird in Deutschland nur für die Verschlüsselung der Todesursachen eingesetzt. In den statistischen Landesämtern wird die Kodierung der auf den Todesbescheinigungen eingetragenen Diagnosen anhand von Band 1-3 der ICD-1o der WHO vorgenommen und ein Grundleiden pro Sterbefall in die Todesursachenstatistik eingetragen. Die rein manuelle Kodierung wird in den letzten Jahren zunehmend durch eine elektronische Kodierung mit der Kodiersoftware Iris ergänzt bzw. ersetzt [DIMDI, 2014c]. Hierbei handelt es sich um eine Software, die von einer internationalen Gruppe erstellt und gepflegt wird. Sie ist in vielen Ländern im Einsatz und hat zum Ziel, die Kodierung der Todesfälle international weiter zu standardisieren und manuell generierte Abweichungen durch unterschiedliche Kodiererfahrung zu vermeiden. Die Software basiert auf komplexen Entscheidungstabellen, in denen die unterschiedlichen, in einem Totenschien enthaltenen ICD10-Kodes anhand der WHO-Regeln zueinander in Bezug gesetzt werden.

\subsubsection{Abrechnung}

Zentraler Treiber für die Weiterentwicklung der ICD-10-GM ist die Abrechnung von medizinischen Leistungen. Die Abrechnung stationärer Leistungen nach 
5 Weiterentwicklung internationaler Terminologien und Klassifikationen und ihre Anwendung in Deutschland am Beispiel der ICD

G-DRG [InEK, 2007-2014a] und nach dem in Entwicklung befindlichen PEPPSystem [InEK, 2007-2014b] erfolgt auf Basis der ICD-10-CM. Hierbei erfolgt die Zuordnung eines Behandlungsfalls zu der entsprechenden Fallpauschale nach der Hauptdiagnose, die mittels ICD-10-GM übermittelt wird.

Auch in der ambulanten Abrechnung spielt die ICD-10-GM eine wichtige Rolle. Neben den entsprechenden Einträgen zur Behandlung gemäß EBM (Einheitlicher Bewertungsmaßstab) [KBV, 2014] muss die behandelte Diagnose mittels ICD-10-GM verschlüsselt sein.

\subsubsection{Morbiditätsorientierter Risikostrukturausgleich}

Bis 2009 wurde der sog. Risikostrukturausgleich zwischen den Krankenkassen durchgeführt. Hierbei wurden u.a. Ausgaberisiken nach Alter, Geschlecht und Erwerbsminderungsstatus für die Versicherten der einzelnen Kassen ermittelt und anhand dieser Risiken ein finanzieller Ausgleich zwischen den Kassen durchgeführt.

Seit 2009 wird über den Gesundheitsfonds der sog. morbiditätsorientierte Risikostrukturausgleich durchgeführt. Dabei fließen alle Einnahmen der Krankenkassen zunächst in den Gesundheitsfonds und werden dann von dort auf die einzelnen Kassen verteilt. Ausschlaggebend für die Höhe der den einzelnen Kassen zugewiesenen Gelder ist neben Alter und Geschlecht der Gesundheitszustand der Versicherten der jeweiligen Kasse. Dabei werden als Indikator für den Gesundheitszustand u.a. die mittels ICD-10-GM dokumentierten ambulanten und stationären Behandlungsdiagnosen genommen [Bundesversicherungsamt, 2014].

\subsubsection{Qualitätsberichte und Qualitätssicherung}

Gemäß gesetzlicher Regelung müssen alle Krankenhäuser, die ihre Leistungen gegenüber Krankenkassen abrechnen, in zweijährigem Rhythmus Qualitätsberichte [G-BA, 2014a] veröffentlichen. In diesen Berichten werden die behandelten Diagnosen gemäß ICD-10-GM angegeben.

Auch die Qualitätssicherung verwendet ICD-10-GM-Kodes. In der externen sektorübergreifenden Qualitätssicherung [G-BA, 2014b] z.B. lösen Schlüsselkodes die jeweiligen QS-Verfahren aus. Qualitätssicherungsrichtlinien des Gemeinsamen Bundesausschusses definieren über Listen von ICD-10-GM-Kodes den jeweiligen Geltungsbereich.

\subsubsection{Morbiditätsstatistik}

In Deutschland wird schon seit langem eine Krankenhausstatistik geführt, in der anhand von ICD-10-GM-kodierten stationären Behandlungsdaten die 
B Der fachliche Hintergrund: Terminologien und Ordnungssysteme in der

Patientenversorgung, medizinischen Forschung und Gesundheitswirtschaft

Anzahl der Behandlungsfälle je Diagnose ausgewiesen wird [GBE, 2014]. Die ambulanten Behandlungsdaten waren bisher nicht gemeinsam mit den stationären Daten verfügbar, sie wurden nur für die Zwecke des morbiditätsorientierten Risikostrukturausgleichs zusammengeführt. Seit Februar 2014 sind im Informationssystem Versorgungsdaten [DIMDI, 2014b] ambulante und stationäre Behandlungsdaten nun erstmals zusammen verfügbar und können unter streng kontrollierten Bedingungen ausgewertet werden.

\subsubsection{Integration der ICD-10 in IT-Systeme}

Die jährlich revidierte ICD-10-GM-Fassung wird von vielen Softwareentwicklern und Anwendern in IT-Systeme eingespielt. Hierzu werden die standardisierten Formate vom DIMDI verwendet. Dem einzelnen Endanwender stehen die Klassifikationen dann direkt z.B. in Systemen zur Führung elektronischer Patientenakten zur Verfügung. Aber trotz zunehmender Digitalisierung gibt es noch immer mehrere Verlage, die die Klassifikationen in Buchformat drucken, und das in nicht unerheblicher Auflage.

Alle oben genannten Anwendungsfelder verwenden die ICD-1o in elektronischem Format und sind somit mit den gleichen Problemen der technischen Einbindung von Formaten konfrontiert, die primär gar nicht für die elektronische Weiterverarbeitung angelegt waren. Auch ist eine Vernetzung der ICD10 mit Terminologien nicht einfach auf elektronische Weise zu lösen. Zwar gibt das DIMDI mit dem umfangreichen alphabetischen Verzeichnis und der daraus erstellten Alpha-ID [DIMDI, 2014a] für die elektronische Weiterverarbeitung eine anwendungsbezogene Terminologie heraus, die für den deutschen Sprachraum die gängigen Diagnosebezeichnungen abdeckt. Allerdings fehlt hier die Vernetzung zu internationalen Terminologie-Systemen, die Anwendung ist somit auf den deutschen Sprachraum begrenzt. Eine standardisierte Weitergabe von Informationen unterhalb des Detailgrades der ICD-10-GM anhand dieser Alpha-ID ist somit nur zwischen Anwendern in Deutschland und der deutschsprachigen Schweiz sinnvoll. Hierfür hat die ICD-10 auch international keine Lösung parat.

Daher besteht die große Hoffnung, dass die Probleme und Hürden, die seit Jahren auf technischer Basis bestehen, mit der ICD-11 gelöst werden können.

\subsection{Wandel der Weiterentwicklung}

In den ersten Weiterentwicklungskonferenzen vor ca. 150 Jahren trafen sich wenige Experten und stimmten einer kleinen Liste von Todesursachen zu bzw. veränderten sie handschriftlich. Für die Überarbeitung zur ICD-1o durch die Weltgesundheitsorganisation (WHO) dagegen waren bereits viele verschiedene Arbeitsgruppen im Einsatz und die Inhalte wurden mit Experten aus der 
5 Weiterentwicklung internationaler Terminologien und Klassifikationen und ihre Anwendung in Deutschland am Beispiel der ICD

ganzen Welt abgestimmt. Die Weiterentwicklung erfolgte bereits in elektronischem Format, jedoch wurde dafür noch nicht vollumfänglich das Potenzial der damals verfügbaren Softwareanwendungen ausgeschöpft. So wurde z.B. das Alphabet zur ICD-1o bei der WHO nicht primär auf Datenbankbasis erstellt, das systematische Verzeichnis wurde in Form von Text-Dateien gepflegt, die eine elektronische Weiterverarbeitung in anderen IT-Systemen erschweren, sei es durch fehlende Strukturierung, sei es durch Einschränkungen durch fest vorgegebene Formate.

Bei der Anwendung der ICD-1o stößt man durch diese technischen Einschränkungen immer wieder auf Probleme. Das DIMDI hat bereits in den goer-Jahren versucht, durch die Bereitstellung der deutschsprachigen Klassifikationen in standardisierten elektronischen Formaten die Verwendung der Klassifikationen in den verschiedenen IT-Systemen zu ermöglichen. Neben einfach strukturieren Text-Dateien wurde u.a. auch eine datenbankfähige SGML-Fassung [W3, 1995-2004] der Klassifikationen für die Anwender entwickelt. Auch die englischsprachige ICD-10 wurde durch das DIMDI für die WHO in diese verschiedenen Formate überführt.

\subsubsection{Technische Basis der ICD-10}

Die SGML-Fassung stellte die Grundlage der deutschen ICD-1o dar, die ab Ende der goer Jahre an die Belange des deutschen Gesundheitssystems angepasst wurde. Das SGML-Format erwies sich allerdings als zu komplex und konnte sich international nicht durchsetzen. Deshalb wurde von der Universität Nimwegen zusammen mit dem DIMDI und der WHO ab 2003 ein Standard für Klassifikationen auf Basis von XML erarbeitet (ClaML - Classification Markup Language), der nach vielen Diskussions- und Adaptationsschritten und nach der 2007 erfolgten Anerkennung als CEN-Norm schließlich im Jahr 2013 als finaler ISO-Standard [ISO, 2013] akzeptiert wurde. Doch auch dieser Standard ist nicht perfekt. Auch wenn in weiten Teilen die nötigen Formatinformationen für die Druckdateien aus dem Standard herausgehalten werden konnten, so ist doch zu erkennen, dass die ICD-10, wie auch viele andere Klassifikationen, die in die Entwicklung des Standards eingeflossen sind, mit den Limitierungen von primär Textdatei-entwickelten Klassifikationen behaftet sind. So ist z.B. die Darstellung von komplexen Tabellen, wie in der ICD-1o von der WHO vorgesehen, nur durch Behelfslösungen im Standardformat ClaML möglich. Auch die Beachtung der Standards für Barrierefreiheit von Dateien spielte offensichtlich bei der Erstellung der ICD-1o keine große Rolle.

\subsubsection{Technische Basis der ICD-11}

Für die nächste Revision soll das nun anders sein. Zurzeit läuft bei der WHO die 11. Revision der ICD [WHO, 2014d]. Laut WHO wird die Verabschiedung 
B Der fachliche Hintergrund: Terminologien und Ordnungssysteme in der

Patientenversorgung, medizinischen Forschung und Gesundheitswirtschaft

durch die World Health Assembly für 2018 erwartet. Technische Basis ist eine Software, die auf Protégé [Stanford Center for Biomedical Informatics Research, 2014] aufsetzt und für die Belange der ICD-Revision modifiziert wurde. Im Rahmen der Revision wurde diese Software, das sog. iCAT (international Collaborative Authoring Tool) [iCAT] immer weiter ergänzt, und es ist nun möglich, neben der Weiterentwicklung der Inhalte auch eine verknüpfte Pflege der dazugehörigen Terminologie durchzuführen, die dann zur Generierung des Alphabetes verwendet werden soll. Dieses soll aber nur insoweit innerhalb des iCATs gepflegt werden, als es zwingend für die Klassifikationsanwendung nötig ist.

Darüber hinaus ist vorgesehen, dass die ICD-11 bereits während der Revision mit weiteren Terminologien und Nomenklaturen verknüpft werden kann, so z.B. mit SNOMED-CT [IHTSDO, 2014]. Auch andere Klassifikationssysteme, wie die von der WHO herausgegebene Internationale Klassifikation der Funktionsfähigkeit, Behinderung und Gesundheit (ICF) [WHO, 2014e], sollen direkt durch Verknüpfungen innerhalb der Datenbank eingebunden werden. Durch eine umfangreiche Datenbasis in dem sog. „Foundation Layer“ (der zugrundeliegenden relationalen Datenbank) soll die Generierung von strukturell und inhaltlich konsistenten sog. „Linearizations“ (auf den jeweiligen Zweck zugeschnittenen Anwendungsversionen) erfolgen (s. Abb. 2). Die Verknüpfung mit anderen bestehenden Systemen hat den Charme, dass eine doppelte Vorhaltung und Pflege von Inhalten vermieden werden kann. Zusätzlich sieht der „Foundation Layer“ Datenfelder für unterschiedliche Sprachversionen vor. Eine Übersetzung der Klassifikation soll also direkt in der gleichen Datenbank durchgeführt und vorgehalten werden, so dass die verschiedenen Sprachversionen der ICD-11 immer alle auf gleichem Stand sein werden.

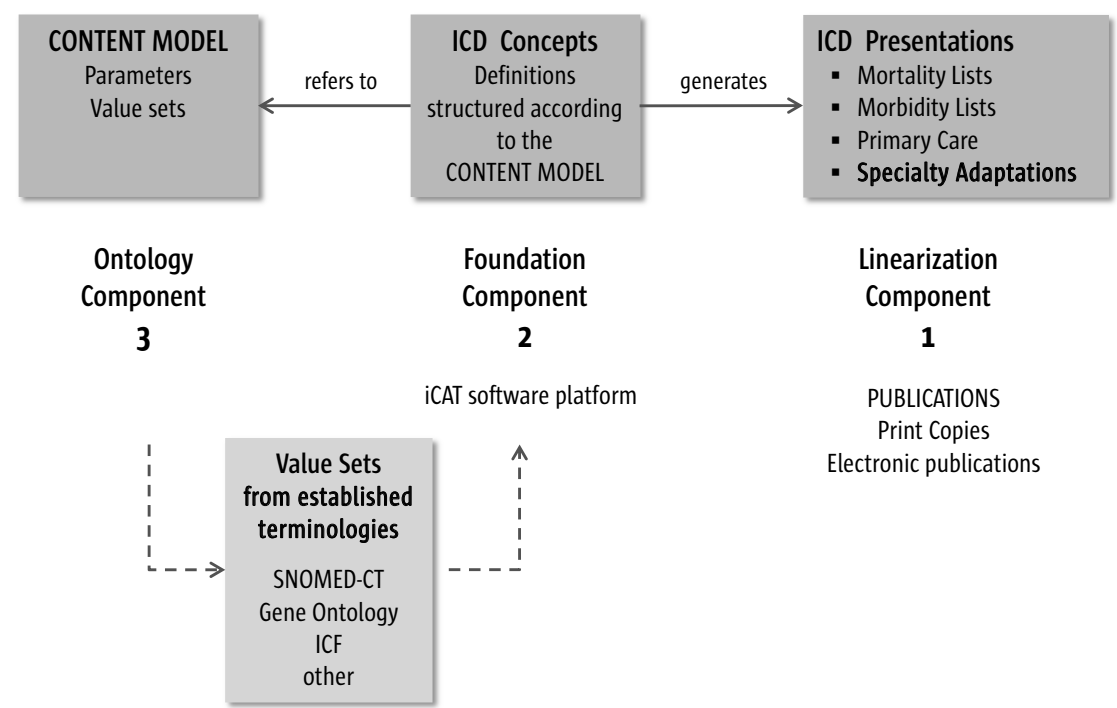

Abb. 2 Das 3-Komponentenmodell der ICD-11 [WHO, 2014a] 


\subsubsection{Inhaltliche Weiterentwicklung}

\section{ICD-10}

Auch inhaltlich hat die ICD-10 im Laufe der letzten 20 Jahre einige Ergänzungen erfahren. Die WHO-Fassung ICD-10-WHO wird im 3-Jahreszyklus mit größeren Updates, die statistische Relevanz haben könnten, auf einen neuen Stand gebracht, jedes Jahr werden kleinere Fehler korrigiert oder kleine Änderungen vorgenommen. Die modifizierte deutsche Fassung ICD-10-GM für die Morbiditätskodierung wird im Jahreszyklus aktualisiert. Hier fließen nicht nur die WHO-Aktualisierungen ein, sondern es werden auch Änderungen umgesetzt, die spezifisch für das deutsche Gesundheitswesen erforderlich sind. Diese können in dem sog. Vorschlagsverfahren des DIMDI [DIMDI, 2014d] von jedermann vorgeschlagen werden; bei Unterstützung durch die Fachgesellschaften und die Selbstverwaltung werden sie entsprechend in der Klassifikation umgesetzt. Änderungsvorschläge aus dem deutschen Vorschlagsverfahren werden bei entsprechender Relevanz auch in das Aktualisierungsverfahren der WHO eingebracht.

Diese Art der Aktualisierung hilft, die Klassifikation auf dem aktuellen medizinischen Stand zu halten. Allerdings stößt das Verfahren manchmal an seine Grenzen, insbesondere wenn sich grundlegende Änderungen im Verständnis von Krankheiten oder Krankheitsgruppen ergeben. Hier kann die Struktur der Klassifikation und die begrenzte Anzahl der Kodes nicht immer die ideale Abbildung der gewünschten Änderung ermöglichen.

Durch die komplexe Verwendung der Klassifikation ist die Änderung von Inhalten der Klassifikation auch nicht immer opportun. Ist für einen bestimmten Einsatzbereich eine Änderung nötig, so kann dies an anderer Stelle zu erheblichen Verwerfungen bei der Implementierung führen. Deshalb werden Änderungen in Deutschland immer erst gründlich geprüft und abgestimmt, bevor sie umgesetzt werden. Dies führt dazu, dass komplexe Änderungen nicht immer innerhalb eines Jahres erfolgen, sondern durchaus bis zur Umsetzung auch mal mehrere Jahre benötigen.

\section{ICD-11}

Durch die 11. Revision will man diese Beschränkungen nun beseitigen und die Klassifikation neu aufstellen. Dabei soll auch ausreichend Spielraum für spätere Aktualisierungen vorgesehen werden.

Viele Bereiche der ICD-1o sind mittlerweile medizinisch überholt und es gibt einige Bereiche, in denen innerhalb der ICD-1o keine befriedigende Lösung gefunden werden konnte. Um alle Belange der Anwender zu berücksichtigen und möglichst viele Experten in den Revisionsprozess einzubinden, hat die WHO das derzeit laufende Verfahren breit aufgestellt. Es wurden diverse Fachgruppen berufen, die sich den Inhalt des jeweiligen Fachbereiches vorgenom- 
B Der fachliche Hintergrund: Terminologien und Ordnungssysteme in der

Patientenversorgung, medizinischen Forschung und Gesundheitswirtschaft

men haben, um ihn zu überarbeiten. Je nach Fachbereich ist dies in mehr oder weniger umfangreicher Art und Weise durchgeführt worden oder auch noch in Arbeit (s. Abb. 3). Diese Änderungen befinden sich nun in einem Stadium, in dem die Kodes auf Detailgenauigkeit und Abgrenzbarkeit gegeneinander überprüft werden müssen. Hier sollen nun die Querschnittsarbeitsgruppen zum Einsatz kommen, die sich aus Klassifikationsexperten aus verschiedenen Ländern und Anwendungsbereichen zusammensetzen.

Aber nicht nur durch die Einbindung von Expertengruppen will die WHO sich Anregungen holen. Auch die breite Öffentlichkeit soll eingebunden werden

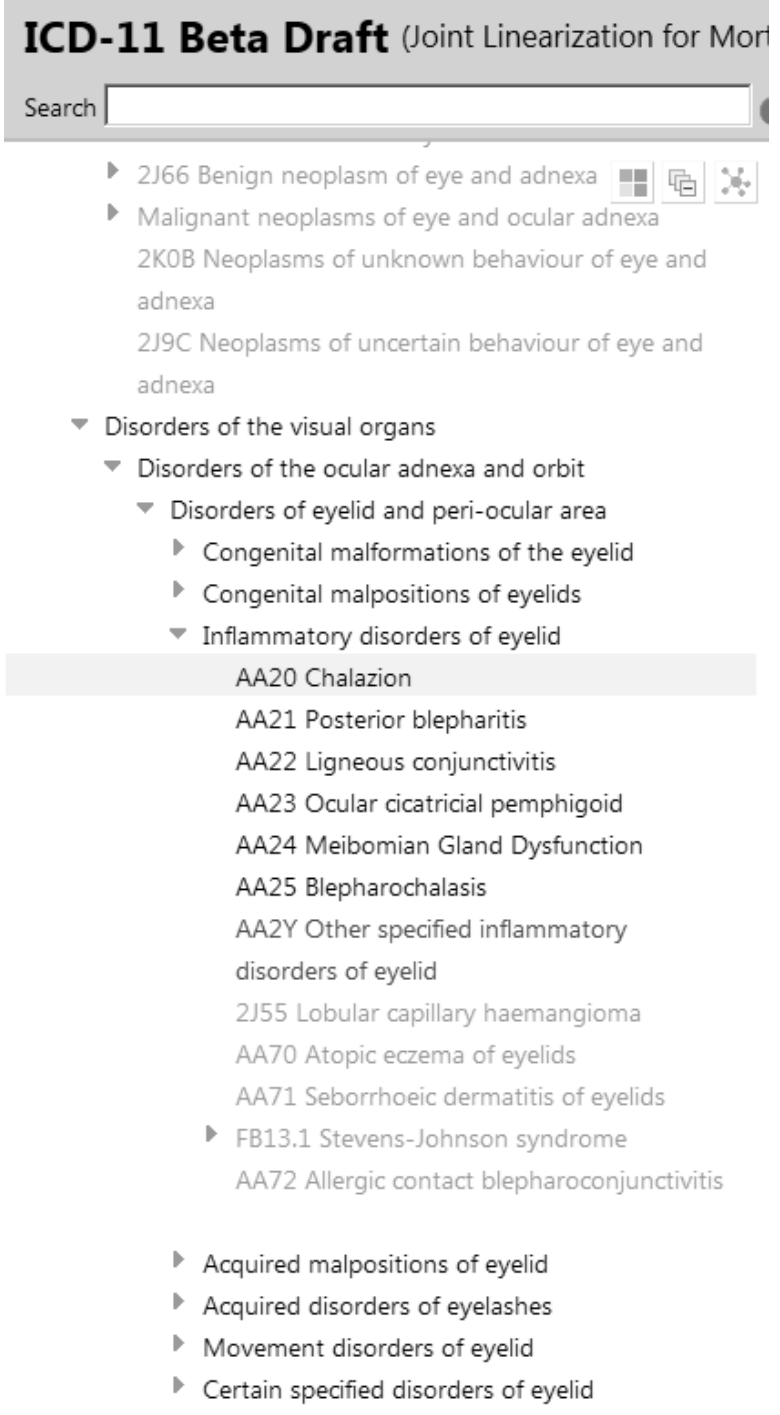

Abb. 3 Kleiner Ausschnitt aus den Krankheiten des Auges der ICD-11 [WH0, 2014c] 
5 Weiterentwicklung internationaler Terminologien und Klassifikationen und ihre Anwendung in Deutschland am Beispiel der ICD

und es wurden Online-Werkzeuge geschaffen, mit denen jeder Interessierte Änderungsvorschläge machen, Änderungen kommentieren oder auch zu klassifikatorischen Strukturen Stellung nehmen kann [WHO, 2014C].

Dieses Unterfangen ist sehr komplex, und die komplette Umstrukturierung der technischen Werkzeuge während des Revisionsprozesses hat die Abläufe weiter verkompliziert. So ist aktuell noch kein befriedigender Zustand der ICD-11 erreicht, der für den Einsatz geeignet ist. Hier soll in den kommenden beiden Jahren noch Einiges passieren, und auch die Vervollständigung des teilweise noch fehlenden Inhaltes soll möglichst zeitnah erfolgen. So ist zum Beispiel die vorgesehene Verlinkung zu anderen Klassifikationen oder Terminologien wie z.B. ICF oder SNOMED CT noch nicht weit fortgeschritten. Hier macht aber der Nutzen einer solchen Verlinkung nur Sinn, wenn diese auch vollständig ist.

Auch bedingt die Komplexität der ICD-11 einen deutlich erhöhten Aufwand bzgl. der Aktualisierung. Dies bezieht sich nicht so sehr auf die technischen Funktionalitäten als eher auf die inhaltlichen Komponenten wie die Kode-Inhalte und die damit verknüpften Zusatzinformationen. Diese Änderungen können nur durch menschliche intellektuelle Leistung erfolgen und bedingen daher auch in Zukunft einen hohen personellen Aufwand. Es bleibt abzuwarten, wie dieses Dilemma in der noch verbleibenden Zeit des Revisionsverfahrens gelöst werden kann und was diesbezüglich für die Zeit nach der Verabschiedung der ICD-11 durch die World Health Assembly geplant wird.

\subsubsection{ICD-11 im Test-Einsatz}

Die WHO möchte schon frühzeitig den Einsatz der ICD-11 in den Anwenderländern testen und hat deshalb innerhalb des Revisionsprozesses eine Phase von Feldtestungen [WHO, 2014b] vorgesehen. Anhand von definierten Protokollen sollen der Inhalt und die technische Struktur im Einsatz erprobt werden. Hiervon erhofft sich die WHO, zielgerichtet weitere Anpassungen vornehmen zu können, bevor die Klassifikation verabschiedet wird.

\subsubsection{ICD-11 in der Routine}

Ob die ICD-11 für den Routineeinsatz geeignet sein wird, kann man zum jetzigen Zeitpunkt noch nicht beurteilen. Klar ist aber schon jetzt, dass der Umstieg von der ICD-10 auf die ICD-11 in den Anwendungsländern mit einem deutlich höheren Aufwand verbunden sein wird als der Umstieg von der ICD-9 auf die ICD-10.

Neben den umfangreichen Anwenderschulungen, die vor und bei Beginn des Einsatzes zu erwarten sind, ist auch die Umstellung einer Vielzahl von IT-Systemen nötig. Da diese fest vernetzt im jeweiligen gesamten Gesundheitswesen 
B Der fachliche Hintergrund: Terminologien und Ordnungssysteme in der Patientenversorgung, medizinischen Forschung und Gesundheitswirtschaft

im Einsatz sind, muss dies gut geplant werden und bedarf eines entsprechenden Vorlaufes. Was genau auf die Anwenderländer zukommen wird, bleibt abzuwarten.

Bringt die ICD-11 wirklich die Lösung für all die Probleme, die bei der Anwendung der ICD-1o bestehen? Kann die geplante Vernetzung mit anderen Systemen z.B. eine Erleichterung der Anwendung herbeiführen? Oder wird vielleicht durch die ICD-11 sogar eine höhere Patientensicherheit erzielt?

Das alles sind Fragen, die zum jetzigen Zeitpunkt noch nicht beantwortet werden können. Fakt ist aber, dass die 11. Revision viele Möglichkeiten der digitalen Vernetzung aufgegriffen hat und versucht, die ICD damit auf den aktuellen Stand von medizinischem Fachwissen und IT-Systemen zu bringen. Ob sich das Ergebnis als praxistauglich erweist, wird man sehen.

\section{Referenzen}

[Bundesversicherungsamt, 2014] Bundesversicherungsamt. Risikostrukturausgleich. http://www.bundesversicherungsamt.de/risikostrukturausgleich.html Letzter Zugang: 2014-09-08.

[DIMDI, 1952] DIMDI. ICD 6 („Ost“). http://www.dimdi.de/dynamic/de/klassi/downloadcenter/icd-vorrevisionen/icd-6/Letzter Zugang: 2014-09-08.

[DIMDI, 2013] DIMDI. ICD-10-GM - Versionsverlauf. http://www.dimdi.de/static/de/klassi/icd-10-gm/historie /gueltigkeit/index.htm Letzter Zugang: 2014-09-08.

[DIMDI, 2014a] DIMDI. Alpha-ID - Identifikationsnummer für Diagnosen. http://www.dimdi.de/static/de/ klassi/alpha-id/index.htm Letzter Zugang: 2014-09-04.

[DIMDI,2014b]DIMDI.InformationssystemVersorgungsdaten(Datentransparenz).http://www.dimdi.de/static/ de/versorgungsdaten/index.htm Letzter Zugang: 2014-09-08.

[DIMDI, 2014C] DIMDI. Iris Institute. www.iris-institute.org Letzter Zugang: 2014-09-08.

[DIMDI, 2014d] DIMDI. Vorschlagsverfahren zur Pflege von OPS und ICD-10-GM für Zwecke des G-DRG-Systems. http://www.dimdi.de/static/de/klassi/icd-10-gm/vorschlagsverfahren/index.htm Letzter Zugang: 2014-09-08.

[G-BA, 2014a] G-BA. Qualitätsbericht der Krankenhäuser. https://www.g-ba.de/institution/themenschwerpunkte/qualitaetssicherung/qualitaetsbericht/? Letzter Zugang: 2014-09-08.

[G-BA, 2014b] G-BA. Sektorenübergreifende Qualitätssicherung. https://www.g-ba.de/institution/ themenschwerpunkte/qualitaetssicherung/sektorenuebergreifend/Letzter Zugang: 2014-09-08.

[GBE, 2014] GBE. Gesundheitsberichterstattung des Bundes: DRG-Statistik. http://www.gbe-bund.de/gbe10/ abrechnung.prc_abr_test_logon?p_uid=gasts $\& p_{-}$aid $=0 \& p \_k n o t e n=F I D \& p \_s p r a c h e=D \& p \_s u c h s-$ tring=10966::drg Letzter Zugang: 2014-09-08.

[iCAT, iCAT. ICD Collaborative Authoring Tool. http://icat.stanford.edu/Letzter Zugang: 2014-09-08.

[IHTSD0, 2014] IHTSDO. SNOMED CT. http://www.IHTSD0.org/SNOMED-ct Letzter Zugang: 2014-08-12.

[InEK, 2007-2014a] InEK. G-DRG-System 2014. http://www.g-drg.de/cms/G-DRG-System_2014 Letzter Zugang: 2014-09-08.

[InEK, 2007-2014b] InEK. PEPP-Entgeltsystem 2014. http://www.g-drg.de/cms/PEPP-Entgeltsystem_2014 Letzter Zugang: 2014-09-08.

[ISO, 2013] ISO. ISO 13120:2013 Health informatics - Syntax to represent the content of healthcare classification systems - Classification Markup Language (ClaML). http://www.iso.org/iso/home/store/catalogue tc/catalogue_detail.htm?csnumber=52952 Letzter Zugang: 2014-09-08.

[KBV, 2014] KBV. Einheitlicher Bewertungsmaßstab (EBM). http://www.kbv.de/html/ebm.php Letzter Zugang: 2014-09-08. 
5 Weiterentwicklung internationaler Terminologien und Klassifikationen und ihre Anwendung in Deutschland am Beispiel der ICD

[Stanford Center for Biomedical Informatics Research, 2014] Stanford Center for Biomedical Informatics Research. protégé - A free, open-source ontology editor and framework for building intelligent systems. http://protege.stanford.edu/Letzter Zugang: 2014-09-08.

[W3, 1995-2004] W3. Overview of SGML Resources. http://www.w3.org/MarkUp/SGML/ Letzter Zugang: 2014-09-08.

[WH0, 2010] WHO. ICD-10 Version: 2010. http://apps.who.int/classifications/icd10/browse/2010/en Letzter Zugang: 2014-09-08.

[WHO, 2014a] WHO. Content Model. http://apps.who.int/classifications/icd/revision/contentmodel/en/ index.htm/ Letzter Zugang: 2014-09-08.

[WHO, 2014b] WHO. Field Tests. http://www.who.int/classifications/icd/revision/fieldtests/en/Letzter Zugang: 2014-09-08.

[WH0, 2014c] WHO. ICD-11 Beta Draft (Joint Linearization for Mortality and Morbidity Statistics). http://apps. who.int/classifications/icd11/browse/l-m/en Letzter Zugang: 2014-09-08.

[WHO, 2014d] WHO. The International Classification of Diseases 11th Revision is due by 2017 http://www.who. int/classifications/icd/revision/en/Letzter Zugang: 2014-09-04.

[WHO, 2014e] WHO. International Classification of Functionning, Disability and Health (ICF). http://www.who. int/classifications/icf/en/Letzter Zugang: 2014-09-08. 